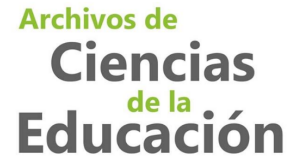

Archivos de Ciencias de la Educación ISSN: 2346-8866

revistaarchivos@fahce.unlp.edu.ar

Universidad Nacional de La Plata Argentina

\title{
El pensamiento de Paulo Freire en tiempo presente. Conferencia de inauguración de la Cátedra Libre Paulo Freire de la UNLP, 23 de mayo de 2019, FaHCE, Ensenada
}

\author{
Puiggrós1, Adriana \\ El pensamiento de Paulo Freire en tiempo presente. Conferencia de inauguración de la Cátedra Libre \\ Paulo Freire de la UNLP, 23 de mayo de 2019, FaHCE, Ensenada \\ Archivos de Ciencias de la Educación, vol. 13, núm. 16, 2019 \\ Universidad Nacional de La Plata, Argentina \\ DOI: https://doi.org/10.24215/23468866e071
}

Atribución no comercial compartir igual (CC BY-NC-SA) 4.0 
Dossier: A 50 años de Pedagogía del Oprimido: lecturas en torno al legado de Paulo Freire

\section{El pensamiento de Paulo Freire en tiempo presente. Conferencia de inauguración de la Cátedra Libre Paulo Freire de la UNLP, 23 de mayo de 2019, FaHCE, Ensenada}

Adriana Puiggrós 1

DOI: https://doi.org/10.24215/23468866e071

Viceministra de Educación de la Nación, Argentina

Muchas gracias a la Universidad Nacional de La Plata, mi universidad, a la que quiero muchísimo y con la cual hace muchísimos años tengo una relación ya que hace muchos, muchos años fue el primer espacio universitario donde estudié. Luego, por distintas razones, terminé en la Universidad de Buenos Aires y en la Universidad del Litoral. Realmente que se abra en esta universidad, en esta facultad, en este espacio, en este ámbito donde uno camina y ve estudiantes que hacen deportes, donde se ve que hay un clima de convivencia, es un lugar donde se ve que hay una universidad... que aquí se abra esta Cátedra Libre Paulo Freire en la Argentina es un hecho muy particular y me parece que, en realidad, corresponde. Era aquí, era en esta universidad, en este lugar dónde tenía que abrirse la Cátedra Libre Paulo Freire.

Les voy a contar una anécdota. Yo recuerdo la primera vez que vino Paulo Freire a la Argentina. En 1974 Freire fue invitado por el Ministerio de Educación de la Nación y además vino a la Universidad de Buenos Aires. Por razones de organización, una noche -él se iba en la madrugada siguiente- fuimos a cenar con Freire, un psicólogo -que hace muchos años vive en España- y yo porque él quería comer empanadas y tomar vino. Le encantaban las empanadas. Nos quedamos toda la noche conversando con él y entonces Freire decía: "esto no aguanta, esta situación no aguanta. No hay un clima social suficiente como para que la situación se sostenga". Consideraba necesario que haya una presencia estudiantil -que sí la había, pero de otra naturaleza de la que hay ahora- y él reclamaba algo que nosotros no terminábamos de entender. Decía que hacía falta un cambio cultural que todavía no se había producido. Le discutimos durante toda la noche. La siguiente comunicación que tuve con Freire fue desde México, unos meses después, porque habían intervenido las universidades. Entonces recuerdo que le mandé una carta en la que le decía: "Sí, Paulo, tenías razón, lamentablemente tenías razón".

Freire en ese momento ya estaba en Ginebra. Se había tenido que ir de Brasil con el golpe de Estado en 1964. Había estado preso unos meses y después un movimiento internacional muy importante logró que lo liberaran y se fue a Chile hasta 1969. Y ahí fue a Ginebra, acogido por el Consejo Mundial de Iglesias (CMI) y vivió muchos años en Ginebra. Recién venía conversando con la profesora Elvira Romera ${ }^{2}$ que conoció muy bien todo ese periodo de Freire. Desde el CMI, Freire fue a Guinea Bissau y a Tanzania donde pudo vincularse con sectores populares en diferentes lugares del mundo y compartir su experiencia brasileña de la cual tomó dimensión mucho más universal. Por eso asombra cuando uno ve que un hombre que empezó trabajando durante el gobierno de Getulio Vargas, con un programa de la Alianza para el Progreso en la ciudad de Recife -con sectores pobres inspirados por una vocación cristiana y queriendo acercarse, viendo cómo se podía hacer para acercarse a aquellos que tenían otra cultura, que pensaban distinto y con los cuáles sentía casi una incomunicación- cómo pudo trascender de manera tal que uno hoy puede encontrar en los confines del mundo, en Taiwan, por ejemplo, Institutos Paulo Freire o en la Universidad de California donde se acaba de hacer una compilación de trabajos de autores de diversos lugares del mundo acerca de Paulo Freire. Precisamente a raíz de esta compilación ${ }^{3}$ en dónde escriben sobre Freire franceses, taiwaneses, chinos, rusos, 
ingleses, pensé que Freire finalmente es latinoamericano, y está muy bien que esto saliera al mundo, pero, a ver, ¿de qué manera salimos al mundo? Entonces a mí me gustaría decir, de alguna manera, ¿por qué Freire es latinoamericano? ¿Cómo creció Freire en América Latina?

En primer lugar, creo que Freire fue un hombre del siglo XX. Su pensamiento es del siglo XX. Esto es importante tenerlo en cuenta, los que estamos acá y los que forman parte de la cátedra, tenemos un papel importante: debemos hacer algo así como una traducción de Freire en el siglo XXI. Ya sabemos que la traducción nunca es literal, siempre es reinterpretación, siempre es reescritura. Freire desarrolla su pensamiento en un momento muy particular. Ese momento que yo marcaba con la anécdota inicial, es el momento del Che Guevara, la América Latina del Che. Un momento donde hay un desarrollo muy importante de la Teología de la Liberación, una influencia que llega sobre Freire de pensadores como Emmanuel Mounier o como Jacques Maritain. Es decir, ese cristianismo liberal con toques del existencialismo francés que tiene mucha importancia en el pensamiento de Freire, sobre todo Mounier. Sumada la lectura de Eric Fromm. Entonces en ese marco es que Freire piensa a América Latina. Y es desde ese marco que va a producir su primer libro La educación como práctica de la libertad. Ese es el mundo. Ese no es nuestro mundo. Nuestro mundo ha cambiado mucho. Pero ese mundo, desde ese mundo, es que piensa Freire.

Ahora, ¿cómo era América Latina? ¿cómo pensar a América Latina? ¿cómo se pensaba a América Latina? América Latina, en esa época, se pensaba como unidad. Hubo varias épocas en las cuales existió un pensamiento latinoamericano. Por ejemplo, a principios del siglo XX, había pensamiento latinoamericano. Rubén Darío era un exponente, andaba por toda América Latina, como Alfredo Palacios, viajaban por América Latina. El propio Gabriel Del Mazo y la Reforma de 1918 tenía un pensamiento latinoamericano. El Manifiesto Liminar es pensamiento latinoamericano. Otro momento fuerte fue el momento en el cual se conforma Freire como intelectual, como pensador.

Ahora, estos esfuerzos por construir América Latina -uno muchas veces piensa que hay que hacer un gran esfuerzo por construir América Latina- ¿qué es lo que une América Latina? ¿qué une a los países latinoamericanos? Porque realmente venimos de una historia de balcanización, una historia de dispersión. Quizás el primer golpe a la unidad latinoamericana fue el Tratado de Tordesillas en 1494 que establece el límite entre las tierras portuguesas y las tierras españolas. Este es quizás el primer corte sobre América Latina. Y después tenemos a Inglaterra actuando para dividir. Tenemos episodios más cercanos que nosotros conocemos como la Guerra de la Triple Alianza que hundió a Paraguay quién sabe por cuánto tiempo. Todavía ahí está Paraguay, no puede levantar cabeza. Y después avanzado el siglo XX, después de la Segunda Guerra Mundial, cuando los Estados Unidos pueden poner en juego aquello puesto en práctica en el ejercicio de la presidencia de Monroe. La famosa Doctrina Monroe que nos coloca en el patio de atrás, o sea, en donde los Estados Unidos son nuestros hermanos mayores que nos van a proteger de cualquier tipo invasión. Y luego las numerosas políticas no tendientes a la unidad sino justamente a la separación de América Latina. Por eso, digamos, el poder construir conceptualmente a América Latina es un ejercicio que quizás todos los intelectuales latinoamericanos deberíamos hacer. En particular, los argentinos, porque Argentina por razones históricas, culturales, de inmigración, como todos sabemos, se ha mantenido siempre imaginariamente en un espacio más vinculado con Europa que con América Latina. No hace demasiado tiempo que nos hemos visto volcados a América Latina lamentablemente, por situaciones muy graves, situaciones muy tristes, donde de repente vemos la pobreza del resto de América Latina y decimos que también es la nuestra.

De todas maneras, ha habido acercamientos muy importantes. La reforma constitucional de 1994 en su capítulo sobre los nuevos derechos establece un acápite que reconoce la propiedad y los derechos de los pueblos originarios. Lo cual no solo es importante por los pueblos originarios, sino que es importante como concepto respecto a la prioridad de la identidad latinoamericana respecto a otras identidades. Ahora, yo creo que este tema de las lenguas, de las diferentes lenguas que subsisten y son tapadas por el portugués o por el castellano son un conjunto de lenguas que existen en América Latina. Las lenguas de muchísimos chicos del 
conurbano con quienes los docentes trabajan todos los días, los chicos guatemaltecos, los chicos mexicanos, los chicos ecuatorianos. Son lenguas vivas que apenas empiezan a ser reconocidas.

Este era uno de los problemas que encontraba Paulo Freire: el encontrarse con una pluralidad cultural muy compleja a la cual había que responder de alguna manera. En un primer momento, él trataba de entenderla con la categoría de colonización. Utiliza esta categoría muy en boga en la época. Claro que se le discute mucho si se puede hablar de la colonización latinoamericana en el siglo XX, si no es descolonización o si en realidad es una etapa neocolonial. Pero es una discusión que la podemos dejar para la academia. El hecho concreto es que lo que descubre Freire es que la propia pedagogía tiene un elemento que él llama colonizador. Es desde allí que va a construir la categoría de educación bancaria que realmente es abarcativa casi de la historia de los sistemas educativos modernos. Es decir, la forma en cómo él la usa es abarcativa de los sistemas educativos modernos que de alguna manera funciona como un significante vacío y entonces uno puede proyectar distintas situaciones y es una categoría muy útil. Frente a eso es que se plantea que, si América Latina ha sufrido diversas colonizaciones. El colonizador ha sido el evangelizador que en realidad lo que hizo fue educar masivamente. Las órdenes religiosas produjeron la primera gran campaña de "alfabetización" masiva de la historia. Al mismo tiempo que un masivo disciplinamiento por la vía simbólica. Y también, digamos, la existencia de alternativas.

Yo no sé si Freire tenía conocimiento de un gran ejemplo de que durante la colonia también había alternativas. Pero si no conocía el ejemplo al menos intuía que la alternativa era posible. El gran ejemplo es el Colegio de Santa Cruz de Santiago de Tlatelolco. En el siglo XVI su rector era Fray Bernardino de Sahagún. Vale la pena que alguna vez lean algunos escritos de Sahagún porque son maravillosos. Allí Fray Bernardino lo que intenta es una relación dialógica, intenta que, en la escuela, que era una universidad, convivan indígenas que eran los hijos de los nobles indígenas, con los españoles. Pero el intento, es un intento de trabajo conjunto de dos culturas. Esto es, de alguna manera, lo que Freire se plantea frente a lo que él va a llamar los desharrapados del mundo, a los cuales dedicó su segundo libro, Pedagogía del Oprimido que escribe entre 1967 y 1969 durante su exilio chileno. Se publica por primera vez en 1970, en Montevideo y Nueva York, mientras él ya se encontraba en Ginebra. A él lo que le preocupa es la no relación, la falta de diálogo. Rechaza características o categorías esencialistas que suponen que el diálogo no es posible entre culturas distintas, entre lenguas distintas, que hay un otro que es totalmente ajeno, al cual lo que hay que hacer es enseñarle. Y en ese sentido cuestiona la racionalidad de la cultura occidental. Lo cuestiona no desde una postura irracionalista, no desde una postura sensiblera, sino que lo cuestiona desde una filosofía de la educación crítica que busca otro racionalismo que sea capaz de inscribir al otro.

Así, por ejemplo, él dice que el diálogo es relacional y que nadie tiene la iniciativa absoluta. Ninguno de los dialogantes tiene la iniciativa absoluta. Junto a esto quiero recordar otra cosa que dice Freire para evitar confusiones. Freire nunca niega al educador y tiene que salir muchas veces a advertir cuando la prensa dice que Freire niega al educador. Esa idea de que en algo relacional no hay primacía de uno por sobre el otro quiere decir que niega al educador. En realidad, Freire subraya que tiene que haber un educador y un educando; solamente que el educando también tiene saberes. Cuestión particularmente importante para la época en que vivimos. Ya que la diferencia de saberes entre las generaciones. La tradicional diferencia de saberes entre la generación como la mía y la de los más jóvenes, y la de los niños eran diferencias porcentualmente estables. Hoy han desaparecido, se han trastocado los lugares de los saberes donde el vínculo pedagógico forzosamente tiene que reubicarse. Pero el problema es que se reubique sin que se pierda la convicción de que no es que hay un vaciamiento de saberes por parte de algunas de las generaciones, sino que hay una postura distinta por parte de los sujetos en vínculo pedagógico.

En Freire hay un evento muy importante que es que él vincula la educación con la política. No me refiero a la política partidaria, sino al vínculo político. Hay de alguna manera una comprensión de lo que Foucault ha llamado la microfísica del poder. O sea, la mirada de Freire va al elemento de poder, al elemento político que se produce en el vínculo pedagógico. Lo que nos permite a nosotros pensar la cuestión de la microfísica del 
poder en las instituciones educativas. Tema que es tomado de otra manera, a mi modo de ver profundamente negativa por sus consecuencias en la actualidad, por Iván Illich. Pero quiero subrayar esa interioridad que marca Freire entre educación y política.

$\mathrm{Al}$ mismo tiempo creo que ese sentido que le da a la educación se vincula con la importancia que le da a la conciencia. La conciencia como lugar de esa razón crítica que mencionaba antes. Ese papel del educador que es ayudar al educando a reflexionar y tomar conciencia de su mundo, de su lugar en el mundo y, por lo tanto, a tomar conciencia del conjunto de saberes que lo constituyen y constituyen ese mundo. En ese sentido, es que me parece que actualmente confrontar a Paulo Freire con Iván Illich es un ejercicio interesante. Iván Illich, un sacerdote católico que rompe con la Iglesia Católica y al hacerlo lo hizo también con el conjunto de las instituciones, por eso, tiene una postura anti institucional. A fines de los años ' 60 se establece en Cuernavaca, México y crea el centro CIDOC (Centro Intercultural de Documentación) que es un lugar donde produce con otros la teoría de la desescolarización, es decir, la idea de Illich es que la escuela ya no existe. Y aquí hago un paréntesis para contar una anécdota. Para esa época había un gobierno popular en Bolivia, ese gobierno tenía como base a los docentes. En ese contexto lo invitan a Illich a hablar en el balcón del Palacio Quemado que es el palacio de gobierno en La Paz. Illich dice: "saben que docentes: la escuela es una vieja y gorda vaca sagrada. Así que vuelvan a sus casas, deshagan las escuelas, dejen en libertad para que se eduquen todos como puedan". Hay un libro de Illich que se llama "La escuela, esa vieja y gorda vaca sagrada".

Frente a esto, la posición de Freire es muy distinta. Es una construcción de saber, una posición de construcción del vínculo, no de disolución del vínculo. La posición de Illich lleva finalmente a una postura individualista que tiene un profundo parecido, sino identidad finalmente, con la postura de los fundadores del neoliberalismo como la Escuela Austríaca de Economía con Von Mises, con Friedman, con Hayek. Aquellos que en los años ' 40 se reunieron en Suiza pensaron el neoliberalismo, economistas que lo pensaron y lo pusieron en práctica. Deben haber entendido muy bien hacia donde iba la sociedad para poder armar ese modelo, porque siempre que se arman estos modelos, hay algo que nosotros no podemos ver. Lo cierto es que esta diferencia me parece una diferencia muy importante. Hoy las ideas de Iván Illich son base de un pensamiento irracional, sensiblero, que realmente descalifica el saber. Quiero subrayar que esto no ocurre así en Estados Unidos, por ejemplo. Trump dice muchas barbaridades, muchas cosas tremendas, pero de ninguna manera, aún desde su lugar de nacionalista conservador, va a pensar en deshacer el gran sistema educativo estadounidense o en que haya "homeschooling", la moda de educar a los niños en las casas en lugar de mandarlos a las escuelas. Salvo una porción de gente muy "snob" que prefiere "homeschooling" en vez de mandar a sus hijos a una institución. Yo creo que hay que tener bastante claro que las teorías desescolarizantes que atacan a la escuela, que atacan a la universidad, que están en contra de que el Estado invierta en que grandes sectores sociales lleguen a la universidad, esas teorías anti-institucionales son teorías realmente nefastas y detrás lo que hay es un irracionalismo.

Por eso, retomando a Freire, lo que uno retoma también es otra tradición que es la tradición de Simón Rodríguez, el maestro de Bolívar, que pensó el sistema educativo de América Latina, que pudo imaginar América Latina a principios del siglo XIX, que recorría América Latina. Imagínense en esa época, iba en carro, en barco, en bote de Colombia a Bolivia. En Potosí puso una escuela y la oligarquía de Bolivia lo echó a las afueras de la ciudad. Una vida tremenda, Bolívar le mandaba dinero para que no se muera de hambre y, sin embargo, imaginaba América Latina y pensaba un sistema educativo liberal democrático, es decir, discutiendo con Santander, con los hacedores del Estado colombiano, sobre todo con aquellos que tenían una línea liberal oligárquica. Esa línea de Simón Rodríguez, de Artigas, de Estanislao López. La línea que también es la de Sarmiento en la idea de educación común, no el Sarmiento racista. Y es que sabemos que tenía un lío en la cabeza. Un Sarmiento que discutía con el otro durante toda su vida. El Sarmiento de la educación pública, ese pensamiento argentino a favor del sistema de educación pública. Esa línea es la línea democrático-radicalizada de tantos inspectores. La línea del movimiento reformista de 1918. La línea del peronismo que establece la gratuidad universitaria. La línea del nacionalismo popular en Argentina. La línea de las grandes reformas de 
las últimas décadas. La línea de las reformas kirchneristas. La línea de las nuevas universidades a donde llegan muchísimos estudiantes que son primera generación, ya no sólo de estudiantes universitarios, sino también de secundarios.

Es esa la línea de Yrigoyen, una que se recupera por lo menos en la primer parte del gobierno peronista, una línea que podemos seguir rastreando en el inspector Vergara, en el inspector Ratier, en muchísimos docentes, muchos de ellos en la Universidad Nacional de La Plata. Hay mucho escrito sobre eso, vale la pena buscarlo. ¿Por qué vale la pena buscarlo? Porque casi todos los que están acá si no todos, son educadores, o son estudiantes y están vinculados a la educación. Todos estamos vinculados a la educación. Y si estamos vinculados con la educación, yo creo que vale la pena, es muy importante. Es inminente buscar una filiación, saber cuál es la historia en la que uno se inscribe. Porque a veces uno se siente perdido, se siente perdido en esa sociedad que se va deshaciendo, en esta sociedad en donde de pronto es como si hubiera cambiado el espacio y el tiempo, como si estuviésemos todos sin fuerza de gravedad, donde no sabemos en qué siglo vivimos, no sabemos en dónde estamos, no sabemos a qué institución pertenecemos. Y creo que es algo muy importante encontrar una filiación, buscar esto que pienso, a dónde se pertenece y con qué historia tomo la decisión de inscribirme. Entonces ahí vamos a encontrar que nosotros no nacimos de la nada, sino que somos producto de una historia.

Y este es otro de los elementos muy importante que plantea Paulo Freire, que es la historicidad. La historicidad del educador, la historicidad del educando, por lo tanto, la historicidad del vínculo pedagógico. Cuando nosotros estamos preparando una clase, cuando estamos dando una clase, cuando estamos trabajando como alumnos en una clase, cuando estamos de espectadores, cuando estamos de alumnos frente a un televisor o frente una plataforma informática que nos tiene de alumnos. Cuando ocurren cosas como que el presidente Netflix, viene a la Argentina y después va a varios países de América Latina, al preguntarle cómo es su vínculo con Microsoft, con Apple, el responde "eso no tiene importancia, nuestra preocupación no es esa. Nuestra preocupación son los sueños, estamos ocupados en cómo hacer para llegar a los sueños".

Entonces cuando quieren capturarnos los sueños, cuando hay grandes plataformas poderosas como, por ejemplo, la editorial inglesa Pearson Education que produce la prueba PISA que como todos sabemos es comprada, adquirida por nuestro país porque nos la impone la Organización para la Cooperación y el Desarrollo Económicos (OCDE). Cuando esas grandes plataformas avanzan sobre nuestra educación, sobre nuestra soberanía, avanzan sobre nuestro espacio educativo, avanzan sobre lo que yo a veces tengo la tentación de llamar algo así como la escuela (sinónimo de universidad o institución educativa) como último espacio de la Nación. A veces me aparece esa frase, se las dejo para que la piensen: la escuela como último espacio de la Nación.

Entonces ahí es donde hay dos categorías de Freire fundamentales para retomar. Una es la de "situación límite”. Pensemos qué es una situación límite, pensemos que es lo que hay en una frontera, cuando la situación límite entre dos lenguas que no se entienden, dos culturas que no se entienden, sectores que no se entienden, momentos en los cuales no se encuentra salida. Y la otra categoría importantísima de Paulo Freire es la de "inédito viable". Esta dificultad que tenemos para imaginar, para proyectar nuevas soluciones, nuevas salidas, desde el aula, desde el momento que estamos trabajando, desde las situaciones generales, desde las situaciones políticas, probablemente tengan que ver con que no conocemos nuestra historia, con que no estamos inscriptos en una filiación. Inscribirnos en esta filiación es permitirse avanzar hacia un inédito viable, que es probablemente algo que hace mucho al futuro de todos nosotros. De manera, yo creo que la apertura de esta cátedra es algo así como una apertura hacia el inédito viable y auguro el mejor de los futuros. Me parece que va a ser un lugar de mucha producción, de mucha imaginación, de mucho aporte a la facultad, a la universidad y sin dudas a la educación argentina y latinoamericana. 
Adriana Puiggrós1. El pensamiento de Paulo Freire en tiempo presente. Conferencia de inauguración...

\section{Notas}

1 Doctora en Pedagogía por la Universidad Nacional Autónoma de México. Profesora Honoris Causa de la UNLP y UNT. Fue Diputada Nacional. Es actual Viceministra de Educación de la Nación, Argentina.

2 Elvira Romera es una pedagoga y militante asociada al movimiento ecuménico. Romera formó parte del destacado grupo de intelectuales que hizo conocer a Paulo Freire en Uruguay siendo la primera editora del brasileño como parte de la Editorial Tierra Nueva. Desde la década de 1970 comenzó una intensa trayectoria en la formación desde la perspectiva del pensamiento latinoamericano en Argentina, México, Guatemala, Puerto Rico y en Costa Rica, junto a Francisco Gutiérrez. Su amistad con Adriana se inicia en los años sesenta durante sus estudios en Ciencias de la Educación en la Facultad de Filosofía y Letras de la UBA.

3 Véase: Carlos Alberto Torres (Editor). The Wiley Handbook of Paulo Freire. Wiley-Blackwell, 2019.

\section{BY-NC-SA}

